\title{
Sphere partition functions and the Zamolodchikov metric
}

\author{
Efrat Gerchkovitz, ${ }^{a}$ Jaume Gomis ${ }^{b}$ and Zohar Komargodski ${ }^{a}$ \\ ${ }^{a}$ Weizmann Institute of Science, \\ Rehovot 76100, Israel \\ ${ }^{b}$ Perimeter Institute for Theoretical Physics, \\ Waterloo, Ontario, N2L 2Y5, Canada \\ E-mail: efrat.gerchkovitz@weizmann.ac.il, \\ jgomis@perimeterinstitute.ca, zohar.komargodski@weizmann.ac.il
}

ABSTRACT: We study the finite part of the sphere partition function of $d$-dimensional Conformal Field Theories (CFTs) as a function of exactly marginal couplings. In odd dimensions, this quantity is physical and independent of the exactly marginal couplings. In even dimensions, this object is generally regularization scheme dependent and thus unphysical. However, in the presence of additional symmetries, the partition function of even-dimensional CFTs can become physical. For two-dimensional $\mathcal{N}=(2,2)$ supersymmetric CFTs, the continuum partition function exists and computes the Kähler potential on the chiral and twisted chiral superconformal manifolds. We provide a new elementary proof of this result using Ward identities on the sphere. The Kähler transformation ambiguity is identified with a local term in the corresponding $\mathcal{N}=(2,2)$ supergravity theory. We derive an analogous, new, result in the case of four-dimensional $\mathcal{N}=2$ supersymmetric CFTs: the $S^{4}$ partition function computes the Kähler potential on the superconformal manifold. Finally, we show that $\mathcal{N}=1$ supersymmetry in four dimensions and $\mathcal{N}=(1,1)$ supersymmetry in two dimensions are not sufficient to make the corresponding sphere partition functions well-defined functions of the exactly marginal parameters.

KEYwords: Supersymmetric gauge theory, Extended Supersymmetry, Anomalies in Field and String Theories

ArXiv EPrint: $1405.7271 \mathrm{v} 2$ 


\section{Contents}

1 Conformal manifolds and sphere partition functions $\quad 1$

2 Sphere partition functions of superconformal field theories 5

$3 \mathcal{N}=(2,2)$ theories and supersymmetric two-spheres $\quad 8$

3.1 The $S^{2}$ partition function $\quad 11$

$\begin{array}{ll}3.2 \text { Kähler ambiguity counterterm and } \mathcal{N}=(1,1) \text { supergravity counterterm } & 14\end{array}$

$\begin{array}{lll}3.3 & \text { An explicit regularization } & 16\end{array}$

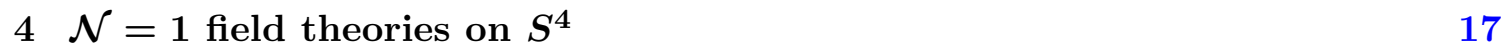

$5 \mathcal{N}=2$ field theories on $S^{4} \quad 19$

5.1 The Kähler potential from the four-sphere 21

\section{Conformal manifolds and sphere partition functions}

Suppose that in a $d$-dimensional CFT, denoted by $p$, there are exactly marginal operators $\left\{O_{i}\right\}$. This means that $\operatorname{dim}\left(O_{i}\right)=d$. In addition, if we deform the theory $p$ by $^{1}$

$$
\frac{1}{\pi^{d / 2}} \int d^{d} x \lambda^{i} O_{i}(x)
$$

then, at least for sufficiently small $\lambda^{i}$, we get a conformal field theory. Thus, in some neighborhood of the reference conformal field theory $p$, there is a family of CFTs. We will refer to this family of CFTs as the "conformal manifold," and denote it by $\mathcal{S}$. This space admits a natural Riemannian metric, the Zamolodchikov metric $g_{i j}(p)$, given by [1]

$$
\left\langle O_{i}(x) O_{j}(0)\right\rangle_{p \in \mathcal{S}}=\frac{g_{i j}(p)}{x^{2 d}},
$$

where $0 \neq x \in \mathbb{R}^{d}$. The couplings $\lambda^{i}$ in (1.1) are coordinates on the conformal manifold $\mathcal{S}$ and the operators $O_{i}$ represent vector fields in $\mathcal{S}$. Thus, under a change of variables $\lambda^{\prime i}=f^{i}\left(\lambda^{j}\right)$ the metric $g_{i j}$ transforms as a symmetric tensor.

Consider an observable $\mathcal{O}$. Suppose we are interested in computing this observable as a function of $\lambda^{i}$. Starting from some reference CFT at $\lambda^{i}=0$, we can thus define

$$
\langle\mathcal{O}\rangle_{\lambda}=\left\langle\mathcal{O} \exp \left[\frac{1}{\pi^{d / 2}} \int d^{d} x \lambda^{i} O_{i}(x)\right]\right\rangle .
$$

\footnotetext{
${ }^{1}$ We choose a normalization that will make later formulae simpler.
} 
In practice, one can expand in the $\lambda^{i}$ around the reference CFT, and define (1.3) in some small neighborhood of $\lambda^{i}=0$ via this expansion. More explicitly,

$$
\langle\mathcal{O}\rangle_{\lambda}=\sum_{k} \frac{1}{k !}\left\langle\mathcal{O}\left(\frac{1}{\pi^{d / 2}} \int d^{d} x \lambda^{i} O_{i}(x)\right)^{k}\right\rangle .
$$

It is very important that (1.4) is ambiguous. Indeed, even if we solve the reference theory at $\lambda=0$ completely, i.e. we know all the correlation functions at separated points, we still need the integrated correlation functions in order to compute (1.4). Integrated correlation functions generically have ultraviolet divergences. They need to be regularized by introducing a cutoff and appropriate counterterms need to be added to the effective action so that the correlation functions are finite in the continuum limit. Therefore, computations of various observables via (1.4) may depend on the regularization scheme, and consequently be subject to ambiguities.

Let us now analyze these ambiguities in some detail. The formalism where the couplings are taken to be dimensionless functions of space rather than constants $\lambda^{i} \rightarrow \lambda^{i}(x)$ allows to easily classify all such ambiguities. Different regularization schemes simply differ by local terms of dimension equal or smaller than $d$ in the effective action. In order to preserve Ward identities, one may need to fine-tune some of the counterterms.

Let us briefly describe a few general pedagogical examples. The finite counterterm

$$
\int d^{d} x \delta \Gamma_{i j}^{k}(x) \lambda^{i}(x) \lambda^{j}(x) O_{k}(x)
$$

generates a contact term in the operator product expansion $O_{i}(x) O_{j}(0) \sim \delta \Gamma_{i j}^{k} \delta^{d}(x) O_{k}(0)$, and affects various integrated correlation functions. The object $\delta \Gamma_{i j}^{k}$ shifts the connection on the conformal manifold $\mathcal{S}$ [2]. In even dimensions, the local counterterm

$$
\Lambda_{U V}^{d / 2} \int d^{d} x C_{i j} \lambda^{i}(x) \lambda^{j}(x) L(x)
$$

where $L$ is a primary operator of $\operatorname{dim}(L)=d / 2$, captures the ultraviolet divergence that appears in computing the correlator $\left\langle L(0) O_{i}(y)\right\rangle_{\lambda}$ in conformal perturbation theory. This term must be carefully tuned to guarantee that $\left\langle L(0) O_{i}(y)\right\rangle_{\lambda}=0$ (for $y \neq 0$ ), which follows from conformal Ward identities. Indeed, to leading order in the expansion (1.4) we get $\int d^{d} x\left\langle L(0) O_{i}(y) O_{j}(x)\right\rangle$. This correlator is infrared finite but has an ultraviolet divergence from the region $x \rightarrow y$, where it behaves as $(x-y)^{-3 d / 2}$. This divergence can be regularized by cutting a little sphere of radius $1 / \Lambda_{U V}$ around $x=y$ and canceled by tuning the local term (1.6). Finally, another example of a counterterm is

$$
\Lambda_{U V}^{d} \int d^{d} x C_{i j} \lambda^{i}(x) \lambda^{j}(x)
$$

with $C_{i j}$ any symmetric tensor. This counterterm accounts for a power divergent piece that appears in evaluating $\langle 1\rangle_{\lambda}$ to second order in conformal perturbation theory.

Our primary interest in this paper lies in CFTs on curved spaces. A conformal field theory can be placed canonically on any conformally-flat manifold, in particular, on $S^{d}$ (in 
this case we can use the stereographic map). Since there are no infrared divergences, we can study the partition function $Z_{S^{d}}$. We can repeat this procedure at any point on the conformal manifold. This provides an interesting probe of the conformal manifold, $Z_{S^{d}}(p)$.

While infrared divergences are absent, ultraviolet divergences remain. As in our discussion above, they are classified by diffeomorphism invariant local terms of dimension $\leq d$ constructed from the background fields $\lambda^{i}$ and the space-time metric $g_{m n}$. One finds the following general answer for the partition function as a function of the point $\lambda^{i}$ on the conformal manifold:

$$
\begin{aligned}
d=2 n: \quad \begin{aligned}
\log Z_{S^{2 n}}= & A_{1}\left(\lambda^{i}\right)\left(r \Lambda_{U V}\right)^{2 n}+A_{2}\left(\lambda^{i}\right)\left(r \Lambda_{U V}\right)^{2 n-2}+\ldots+A_{n}\left(\lambda^{i}\right)\left(r \Lambda_{U V}\right)^{2} \\
& +A\left(\lambda^{i}\right) \log \left(r \Lambda_{U V}\right)+F_{2 n}\left(\lambda^{i}\right) . \\
d=2 n+1: \quad \log Z_{S^{2 n+1}}= & B_{1}\left(\lambda^{i}\right)\left(r \Lambda_{U V}\right)^{2 n+1}+B_{2}\left(\lambda^{i}\right)\left(r \Lambda_{U V}\right)^{2 n-1} \\
& +\ldots+B_{n+1}\left(\lambda^{i}\right)\left(r \Lambda_{U V}\right)+F_{2 n+1}\left(\lambda^{i}\right)
\end{aligned}
\end{aligned}
$$

where $r$ is the radius of $S^{d}$. The power-law divergent terms correspond to counterterms of the type

$$
\Lambda_{U V}^{2 n-2 k+2} \int d^{2 n} x \sqrt{g} A_{k}\left(\lambda^{i}\right) \mathcal{R}^{k-1}
$$

in even dimensions and

$$
\Lambda_{U V}^{2 n-2 k+3} \int d^{2 n+1} x \sqrt{g} B_{k}\left(\lambda^{i}\right) \mathcal{R}^{k-1}
$$

in odd dimensions. Therefore, all the power-law divergent terms in (1.8) and (1.9) can be tuned to zero in the continuum limit.

In even dimensions, the sphere partition function has a logarithmic dependence on the radius (see (1.8)), which cannot be canceled by a local counterterm. It is associated to the Weyl anomaly. The variation of the partition function under a Weyl transformation with parameter $\sigma$ contains $\int d^{2 n} x \sqrt{g} \sigma A\left(\lambda^{i}\right) E_{2 n}$, where $E_{2 n}$ is the Euler density (the other terms in the Weyl anomaly vanish on the sphere, see [3]). However, this violates the Wess-Zumino consistency condition unless $A\left(\lambda^{i}\right)=A$, i.e. $A\left(\lambda^{i}\right)$ is a constant. This is then identified with the usual $A$-type anomaly [3], which is therefore independent of exactly marginal deformations. $^{2}$ This is necessary for its interpretation as a monotonic function under renormalization group flows [1,4-7]. Finally, the function $F_{2 n}\left(\lambda^{i}\right)$ in $(1.8)$ is ambiguous, as it can be removed by the local counterterm ${ }^{3}$

$$
\int d^{2 n} x \sqrt{g} F_{2 n}\left(\lambda^{i}\right) E_{2 n}
$$

In summary, we have shown that the only physical data in the continuum limit of $Z_{S^{2 n}}$ is the A-anomaly, which is independent of the exactly marginal parameters.

\footnotetext{
${ }^{2}$ We thank L. Di Pietro and A. Schwimmer for discussions.

${ }^{3}$ Note that this counterterm does not affect the partition function on the cylinder $S^{d-1} \times S^{1}$. The latter has a natural normalization via radial quantization.
} 
For odd dimensions, absent additional restrictions on the counterterms, we have seen above that all the $B_{i}$ are ambiguous and can be tuned to zero (a logarithmic term is absent because one cannot write an appropriate local anomaly polynomial in odd dimensions.) Importantly, however, there is no counterterm for $F_{2 n+1}\left(\lambda^{i}\right)$ in (1.9). More precisely, the only conceivable dimensionless counterterm would be a gravitational Chern-Simons term

$$
\int C(\lambda) \Omega^{(2 n+1)}
$$

but because of coordinate invariance it cannot depend on the $\lambda^{i}$, i.e. $C(\lambda)=$ constant. Moreover, it has to have an imaginary coefficient due to CPT symmetry. Hence, the real part of $F_{2 n+1}\left(\lambda^{i}\right)$ is an unambiguous physical observable and is calculable in any choice of regularization scheme that preserves coordinate invariance. ${ }^{4}$ It measures the finite entanglement entropy across a $S^{2 n-1}$ in $\mathbb{R}^{2 n, 1}[11] .^{5}$

We now show that $F_{2 n+1}\left(\lambda_{i}\right)$ is constant on the conformal manifold $\mathcal{S}$. Start at an arbitrary point on the conformal manifold and expand to second order

$$
\log Z[\lambda] \simeq \log Z(0)+\frac{\lambda^{i}}{\pi^{d / 2}} \int d^{d} x \sqrt{g}\left\langle O_{i}\right\rangle+\frac{\lambda^{i} \lambda^{j}}{2 \pi^{d}} \int d^{d} x \sqrt{g} \int d^{d} y \sqrt{g}\left\langle O_{i}(x) O_{j}(y)\right\rangle .
$$

Conformal Ward identities on the sphere imply that

$$
\left\langle O_{i}\right\rangle=0 \text {. }
$$

Since this is true at every point on the conformal manifold, we conclude that the continuum conformal field theory sphere partition function is independent of exactly marginal parameters. As we will see, this argument cannot be repeated in even dimensions because there are finite counterterms. Indeed, we will see examples where the sphere partition function does depend on exactly marginal parameters.

We can understand this simplicity of the sphere partition function in odd dimensions from another point of view. The integrated two-point function of exactly marginal operators in the last term of (1.14) is ultraviolet divergent. The singularity arises from the domain where $x \rightarrow y$. The two-point function on the sphere can be obtained from the corresponding two-point function in flat space by a stereographic map. Hence, the integrated two-point function in (1.14) is proportional to

$$
\operatorname{vol}\left(S^{d}\right) \int_{S^{d}} d^{d} y \sqrt{g}\left(\frac{1}{d(0, y)}\right)^{2 d}
$$

\footnotetext{
${ }^{4}$ The imaginary part is more subtle. Only its fractional part is well defined. See, for example, the discussions in [8-10]. We will not comment any further on the imaginary part of $F_{2 n+1}$.

${ }^{5}$ The entanglement entropy provides another way to see that the finite part in even dimensions is ambiguous while in odd dimensions it is physical. Indeed, it is straightforward to write finite local counterterms on the entangling surface of even-dimensional spheres, while in odd dimensions this is impossible. For example, in $d=3$, the entangling surface is a circle, and the finite counterterm $\int_{S^{1}}|\kappa| d l$ is forbidden because the absolute value renders it nonlocal, while without the absolute value symbol it is not consistent with the vacuum being a pure state. We thank S. Pufu for discussing this with us.
} 
where $d(x, y)$ is the $\mathrm{SO}(\mathrm{d}+1)$ invariant distance on the sphere $S^{d}$. This can be regulated by replacing the power $2 d$ in the integrand by $2 d-2 \epsilon$. We can then evaluate the integral in a region where it converges, analytically continuing to the region of interest at the end, to yield

$$
\lim _{\epsilon \rightarrow 0} \frac{\operatorname{vol}\left(S^{d}\right) \operatorname{vol}\left(S^{d-1}\right)}{2^{d+1}} \frac{\Gamma(d / 2) \Gamma(-d / 2+\epsilon)}{\Gamma(\epsilon)} \longrightarrow 0 \quad \text { for } d=2 n+1 .
$$

Therefore the integral vanishes in odd dimensions, and since we have shown that there are no finite counterterms in odd dimensions, the answer computed in any other choice of regularization scheme would yield the same result. The fact that the second term in the expansion (1.14) vanishes holds true around any point on the conformal manifold. This is consistent with the independence of the partition function of exactly marginal couplings. Note that the same regularization scheme yields a nonzero answer in even dimensions. This will be important below.

In summary, we have shown that the (real part of the) finite part of $Z_{S^{2 n+1}}$ in the continuum limit is physical and unambiguous, and is independent of the exactly marginal parameters of the conformal field theory. This is a necessary requirement for the finite part of $Z_{S^{2 n+1}}$ to serve as a candidate monotonic function under renormalization group flows (see $[11-13])$.

\section{Sphere partition functions of superconformal field theories}

Our analysis of the ambiguities of the sphere partition function of a CFT followed from assuming that the partition function can be regulated in a diffeomorphism invariant way. We then classified all the diffeomorphism invariant counterterms and determined their influence on the sphere partition function. Starting in this section, we pursue an analogous analysis for superconformal field theories (SCFTs). Imposing that the sphere partition function of SCFT's can be regulated in a supersymmetric way, we show that the partition function of SCFTs with various amounts of supersymmetry and in various dimensions have (not unexpectedly) a more restricted set of ambiguities. This makes the sphere partition function of such SCFTs interesting, rich observables: the sphere partition functions of some of these theories are known to compute the Kähler potential on the conformal manifold [14, 15].

We now outline the logic of our arguments. Consider a $d$-dimensional SCFT and place it on the sphere by stereographic projection. Such a SCFT is by definition invariant under the corresponding superconformal algebra. As we explained in section 1, however, the partition function of a CFT suffers from ultraviolet divergences that arise when exploring the dependence of the partition function on the conformal manifold $\mathcal{S}$. We now assume that we can regulate the SCFT partition function while preserving the subalgebra of the superconformal algebra that closes into the super-isometries of the $d$-dimensional supersymmetric sphere, which projects out all conformal generators of $S^{d}$. This is the general supersymmetry algebra of a massive supersymmetric theory on the $d$-dimensional sphere.

In the supersymmetric context, the local counterterms that parametrize the ambiguities of the partition function are now supergravity counterterms. In supersymmetric 
theories, the coordinates that parametrize the conformal manifold are the bottom component of an apropriate supersymmetry multiplet. Therefore we must consider the most general dimension $\leq d$ locally supersymmetric couplings of these multiplets to the corresponding supergravity theory. The supergravity theory that must be considered is found by embedding the rigid supersymmetric $d$-dimensional sphere as a supersymmetric background of $d$-dimensional off-shell supergravity, in the framework put forward by [16]. By constructing marginal supergravity counterterms in a given supergravity theory, we can determine the ambiguities and the leftover physical content of the partition function of SCFTs on $S^{d}$.

Let us briefly summarize our main results explained in the rest of the paper. In section 3 we discuss two-dimensional $\mathcal{N}=(2,2)$ SCFTs. It is known that the conformal manifold of such theories is a Kähler manifold, and locally takes a direct product form $\mathcal{S}_{\mathrm{c}} \times \mathcal{S}_{\mathrm{tc}}$, where $\mathcal{S}_{\mathrm{c}}$, and $\mathcal{S}_{\mathrm{tc}}$ are the chiral and twisted chiral manifolds, respectively. These theories can be placed on supersymmetric $S^{2}$ in two different ways while preserving four supercharges $[15,17-19]$. For each choice there is a corresponding two-dimensional $\mathcal{N}=(2,2)$ supergravity theory, which we denote by A and B. Our notation parallels that of $[17,19]$, where the corresponding supersymmetry algebras were labeled by $\mathrm{SU}(2 \mid 1)_{\mathrm{A}}$ and $\mathrm{SU}(2 \mid 1)_{\mathrm{B}}$. Imposing that the SCFT partition function preserves $\mathrm{SU}(2 \mid 1)_{\mathrm{B}}$, we find that there is a finite B-type supergravity counterterm [20]

$$
\int d^{2} \Theta \varepsilon^{-1} R \mathcal{F}\left(\Phi^{i}\right)+c . c .
$$

where $\Phi^{i}$ are chiral superfields, whose bottom components are the coordinates $\lambda^{i}$ in $\mathcal{S}_{\mathrm{C}}, R$ is the supergravity curvature superfield and $\varepsilon^{-1}$ is the supergravity chiral superspace measure. A similar A-type supergravity counterterm can be constructed for a SCFT preserving $\mathrm{SU}(2 \mid 1)_{\mathrm{A}}$ by replacing chiral superfields by twisted chiral superfieds $\Omega$, whose bottom components $\lambda^{i}$ now span $\mathcal{S}_{\text {tc }}$. When these counterterms are evaluated on the supersymmetric $S^{2}$ background they give rise to the following Kähler ambiguities in the partition function

$$
Z_{S^{2}}^{A} \simeq Z_{S^{2}}^{A} e^{-\mathcal{F}\left(\lambda^{i}\right)-\overline{\mathcal{F}}\left(\bar{\lambda}^{\bar{i}}\right)}, \quad Z_{S^{2}}^{B} \simeq Z_{S^{2}}^{B} e^{-\mathcal{F}\left(\lambda^{i}\right)-\overline{\mathcal{F}}\left(\bar{\lambda}^{\bar{i}}\right)} .
$$

If we describe the same $\mathcal{N}=(2,2)$ SCFT in two different ways, with different choices of counterterms, then the partition functions may differ by (2.2). This means that the two-sphere partition functions $Z_{A}$ and $Z_{B}$ are generally not functions, but rather sections: the transitions between different patches may involve holomorphic functions. Our explicit construction of these counterterms gives a microscopic realization of the Kähler ambiguities implied by the proof [15] that the exact two-sphere partition function [17-19] computes the Kähler potential $K$ on the conformal manifold $\mathcal{S}_{\mathrm{c}} \times \mathcal{S}_{\mathrm{tc}}[14,15]$

$$
Z_{S^{2}}^{A}=e^{-K_{\mathrm{tc}}} \quad Z_{S^{2}}^{B}=e^{-K_{\mathrm{c}}} .
$$

For some followup work see e.g. [21, 22].

One well-known way in which the partition function can turn into a section is if there are 't Hooft anomalies. Then, the partition function transforms nontrivially under gauge 
transformations. Ordinary derivatives of the partition function then yield gauge noninvariant quantities (which can be fixed by the Bardeen-Zumino procedure [23], or equivalently, anomaly inflow [24]). Here we encounter a reminiscent situation. The partition function becomes a nontrivial section. As a result, various derivatives of the partition function need to be supplemented with an appropriate connection that for holomorphic derivatives transforms as $\mathcal{A} \rightarrow \mathcal{A}+\partial \mathcal{F}(\lambda)$ under Kähler transformations. It would be nice to understand if the analogy with ordinary anomalies is deeper than these superficial similarities.

In section 3 we also give a new elementary proof of (2.3) that follows from supersymmetry Ward identities and therefore does not require the existence of a Lagrangian description or localization of the partition function. We also show that the two-sphere partition function of $\mathcal{N}=(1,1)$ SCFTs is not a universal, unambiguous observable (other than capturing the A-type anomaly). We construct a local $\mathcal{N}=(1,1)$ supergravity counterterm that changes the finite part of the partition function arbitrarily, similar to what we found for even-dimensional nonsupersymmetric CFTs.

In section 4 we discuss $\mathcal{N}=1$ SCFTs on $S^{4}$. The exactly marginal parameters again give rise to a Kähler manifold [25]. We argue that in this case the partition function on the four-sphere does not have a preferred, unambiguous nontrivial continuum limit. We show this by constructing a finite $\mathcal{N}=1$ superymmetric supergravity counterterm that includes an arbitrary real function $F$ of the moduli $\lambda^{i}$, which now are the bottom components of chiral multiplets $\Phi^{i}$

$$
\int d^{2} \Theta \varepsilon\left(\overline{\mathcal{D}}^{2}-8 R\right) R \bar{R} F\left(\Phi^{i}, \bar{\Phi}^{\bar{i}}\right) .
$$

Evaluated on $S^{4}$, this shifts the finite part of the partition function by an arbitrary function of the moduli.

In section 5 we discuss $\mathcal{N}=2$ SCFTs on $S^{4}$. In this case we argue in two different ways (one of them being supersymmetric localization and the other by using an explicit supersymmetric regularization of the second term in (1.14)) that

$$
Z_{S^{4}}=e^{K / 12},
$$

where $K$ is the Kähler potential on the space of exactly marginal deformations $\mathcal{S}$. That is, the four-sphere partition function becomes physical if the counterterms are restricted to be $\mathcal{N}=2$ locally supersymmetric, and is only subjected to Kähler ambiguities. The partition function is again a section rather than a function, where the transition functions are holomorphic plus anti-holomorphic in the moduli. Note the sign difference of the exponent (2.5) with respect to (2.3).

One can therefore, in principle, use the localization computation on $S^{4}$ [26] to compute the exact Zamolodchikov metric on $\mathcal{N}=2$ conformal manifolds, extending what has been done for two-dimensional $\mathcal{N}=(2,2)$ SCFTs. It would be interesting to see if one can also derive (2.5) by generalizing the argument of [15] (i.e. geometrically deforming the sphere and using the $t t^{*}$ equations [27]) to four dimensions (the generalization of the $t t^{*}$ equations should be along the lines of [28]). One may also try to extend to four dimensions our supersymmetry Ward identity proof in section 3 . It would also be very nice to 
explicitly construct the four-dimensional $\mathcal{N}=2$ supergravity analog of the holomorphic counterterm (2.1), perhaps using the tools of [29, 30]. Finally, it would be interesting to understand whether the conformal manifold of four-dimensional $\mathcal{N}=2$ SCFTs has extra geometric structure beyond Kähler (additional geometric structure is known to exist in $\mathcal{N}=(2,2)$ two-dimensional SCFTs with $c=9$, see e.g. [31-33]) and also [34] for $c=12$.

In this paper we have confined ourselves to discussing conformal field theories and their sphere partition functions. However, some of our results can be relevant for the interpretation of partition functions of gapped theories as well. For example, if we study a massive theory with a gap at the scale $M$ on a manifold with typical scale $R$ much larger than the the scale associated to the gap $R \gg 1 / M$, then the partition function can be organized as a series expansion in $(R M)^{-1}$ with the various terms in the expansion corresponding to local terms in the action for the background fields. Therefore, supergravity counterterms of the type discussed in this paper can be useful to understand the leading terms in the expansion of partition functions of massive supersymmetric theories.

\section{$3 \mathcal{N}=(2,2)$ theories and supersymmetric two-spheres}

Superconformal $\mathcal{N}=(2,2)$ theories in flat two-dimensional space posses a $\mathrm{U}(1)_{\mathrm{V}} \times \mathrm{U}(1)_{\mathrm{A}}$ R-symmetry group. Exactly marginal operators are superconformal descendants of operators in the chiral and twisted chiral rings which carry charge $(2,0)$ and $(0,2)$ under $\mathrm{U}(1)_{\mathrm{V}} \times \mathrm{U}(1)_{\mathrm{A}}$. We can view the coupling constants for these operators as charge $(0,0)$ background chiral superfields and charge $(0,0)$ background twisted chiral superfields, respectively. The conformal manifold of $\mathcal{N}=(2,2)$ superconformal field theories is Kähler and the Zamolodchikov metric takes a factorized form $\mathcal{S}_{\mathrm{c}} \times \mathcal{S}_{\mathrm{tc}}$ : locally it is the sum of the metric in the chiral and twisted chiral directions. ${ }^{6}$

Such conformal field theories can be placed on $S^{2}$ by a stereographic transformation. The spinors that generate the $\mathcal{N}=(2,2)$ superconformal transformations are conformal Killing spinors $\epsilon$ and $\tilde{\epsilon}$, which satisfy

$$
\nabla_{m} \epsilon=\gamma_{m} \eta, \quad \nabla_{m} \tilde{\epsilon}=\gamma_{m} \tilde{\eta} .
$$

Here, $\gamma_{m}$ are the curved space Dirac matrices, and we define the projection operators $P_{ \pm}=\frac{1}{2}\left(1 \pm \gamma_{3}\right)$ onto the Weyl spinors, e.g. $P_{ \pm} \zeta=\zeta_{ \pm}$. The charges of the spinors under

\footnotetext{
${ }^{6}$ One can derive this as follows. Regardless of supersymmetry, in any two-dimensional CFT, promoting the exactly marginal parameters $\lambda^{i}$ to background fields, one has an admissible conformal anomaly [35]

$$
\delta_{\sigma} W\left[g_{m n}, \lambda^{i}\right]=\int d^{2} x \sqrt{g} \sigma\left(g^{m n} C_{k l}(\lambda) \partial_{m} \lambda^{k} \partial_{n} \lambda^{l}\right)
$$

where we omitted the usual A-type anomaly, as we have already discussed it in the introduction. $C_{k l}$ is some symmetric tensor that depends on the exactly marginal parameters. $C_{k l}$ is proportional to the Zamolodchikov metric on the space of CFTs. This is because the momentum space two-point function of exactly marginal operators looks like $p^{2} \log \left(p^{2}\right)$, which clearly contains a rescaling anomaly. Such a "nonlinear sigma model" (3.1) is supersymmetrized as usual, by replacing the two-derivative term with a Kähler function for the background superfields. Hence, the total space of exactly marginal couplings is Kähler. Since the top component of the product of a chiral field with twisted chiral fields is a total derivative, this leads to a factorized Zamolodchikov metric.
} 
the $R$-symmetry group are as follows

$$
\begin{array}{ccc} 
& \mathrm{U}(1)_{\mathrm{V}} \mathrm{U}(1)_{\mathrm{A}} \\
\epsilon_{+}, \eta_{-} & 1 & 1 \\
\epsilon_{-}, \eta_{+} & 1 & -1 \\
\tilde{\epsilon}_{-}, \tilde{\eta}_{+} & -1 & 1 \\
\tilde{\epsilon}_{+}, \tilde{\eta}_{-} & -1 & -1
\end{array}
$$

The equations (3.2) have an eight-complex-dimensional space of solutions on $S^{2}$. These furnish four doublets of the $\mathrm{SU}(2)$ isometry group of the two-sphere.

The superconformal algebra on $S^{2}$ can be linearly realized on supermultiplets. For our purposes of analyzing the conformal manifold of $\mathcal{N}=(2,2)$ superconformal field theories, it will suffice to consider a chiral multiplet of charge $\left(-r_{V}, 0\right)$ and a twisted chiral multiplet of charge $\left(0,-r_{A}\right)$. The superconformal transformations of a chiral multiplet are [17]

$$
\begin{aligned}
& \delta \Phi=\tilde{\epsilon} \psi \\
& \delta \psi=i \partial_{m} \Phi \gamma^{m} \epsilon+\tilde{\epsilon} F-i r_{V} \eta \Phi \\
& \delta F=-i \nabla_{m} \psi \gamma^{m} \epsilon+i r_{V} \psi \eta
\end{aligned}
$$

A superconformal invariant can be constructed from the top component of a chiral multiplet of Weyl weight $1\left(r_{V}=-2\right)$

$$
I=\int d^{2} x \sqrt{g} F,
$$

since in this case $\delta F=-i \nabla_{m}\left(\psi \gamma^{m} \zeta\right)$, and the variation integrates to zero.

The twisted chiral multiplet transformations are given by [15]

$$
\begin{aligned}
\delta Y & =\left(\tilde{\epsilon} P_{-}-\epsilon P_{+}\right) \zeta \\
\delta \zeta_{+} & =-P_{+}\left(i \partial_{m} Y \gamma^{m}-G\right) \tilde{\epsilon}+i r_{A} Y \tilde{\eta}_{+}, \\
\delta \zeta_{-} & =P_{-}\left(i \partial_{m} Y \gamma^{m}-G\right) \epsilon-i r_{A} Y \eta_{-}, \\
\delta G & =i \epsilon P_{-} \not \zeta-i \tilde{\epsilon} P_{+} \not \nabla \zeta+i r_{A} \zeta P_{-} \eta-i r_{A} \zeta P_{+} \tilde{\eta} .
\end{aligned}
$$

For a twisted chiral multiplet of Weyl weight one $\left(r_{A}=-2\right)$ the integral of the top component

$$
I=\int d^{2} x \sqrt{g} G
$$

is a superconformal invariant, since $\delta G=i \nabla_{m}\left(\epsilon P_{-} \gamma^{m} \zeta\right)-i \nabla_{m}\left(\tilde{\epsilon} P_{+} \gamma^{m} \zeta\right)$.

The superconformal invariants (3.5)(3.7) represent the exactly marginal operators that are descendants of operators in the chiral and twisted chiral rings. The anti-chiral and antitwisted-chiral transformations and invariants are constructed similarly.

The superconformal superalgebra just described admits interesting "massive subalgebras" with four supercharges. By this we mean that massive $\mathcal{N}=(2,2)$ quantum field theories can be placed on $S^{2}$ while respecting these subalgebras [15, 17-19].

A regularization scheme can be thought of as a deformation of the theory by some massive sector. Hence, in order to understand the possible ways of regularizing the partition 
function $Z_{S^{2}}$ while preserving some supersymmetry, we need to study these massive subalgebras in detail. We will mainly focus on the subalgebras that preserve four supercharges, but will also pay some attention to one subalgebra that preserves two supercharges.

There are two inequivalent massive $\mathcal{N}=(2,2)$ supersymmetry algebras on the twosphere. They are generated by supercharges in the superconformal algebra that close into the $\mathrm{SU}(2)$ isometry of the two-sphere and a $\mathrm{U}(1)$ subgroup of the $\mathrm{U}(1)_{\mathrm{V}} \times \mathrm{U}(1)_{\mathrm{A}}$ superconformal R-symmetry. The algebras that preserve $\mathrm{U}(1)_{\mathrm{V}}$ and $\mathrm{U}(1)_{\mathrm{A}}$ have been denoted by $\mathrm{SU}(2 \mid 1)_{\mathrm{A}}$ and $\mathrm{SU}(2 \mid 1)_{\mathrm{B}}$ in $[17,19]$. They are mapped to each other by the mirror outer automorphism [36] of the $\mathcal{N}=(2,2)$ superconformal algebra.

One can also study massive subalgebras preserving only two supercharges. We do not carry out an exhaustive analysis of this case, but only discuss one example: the massive $\mathcal{N}=(1,1)$ subalgebra $\mathrm{OSp}(1 \mid 2)$, consisting of an $\mathrm{SU}(2)$ doublet of supercharges closing into the $\mathrm{SU}(2)$ isometries of $S^{2}$, thus breaking the $R$-symmetry group completely.

- $\mathrm{SU}(2 \mid 1)_{\mathrm{A}}$. This supersymmetry algebra is obtained by restricting the conformal Killing spinors on the two-sphere (3.2) to obey

$$
\nabla_{m} \epsilon=\frac{i}{2 r} \gamma_{m} \epsilon \quad \nabla_{m} \tilde{\epsilon}=\frac{i}{2 r} \gamma_{m} \tilde{\epsilon}
$$

so that

$$
\eta=\frac{i}{2 r} \epsilon, \quad \tilde{\eta}=\frac{i}{2 r} \tilde{\epsilon} .
$$

In stereographic coordinates, where $d s^{2}=\frac{1}{\left(1+\frac{x^{2}}{4 r^{2}}\right)^{2}} d x^{m} d x^{n} \delta_{n m}$, the complex fourdimensional space of solutions is given by

$$
\begin{gathered}
\epsilon=\frac{1}{\sqrt{1+\frac{x^{2}}{4 r^{2}}}}\left(1+\frac{i}{2 r} x^{m} \Gamma_{m}\right) \epsilon_{0}, \\
\tilde{\epsilon}=\frac{1}{\sqrt{1+\frac{x^{2}}{4 r^{2}}}}\left(1+\frac{i}{2 r} x^{m} \Gamma_{m}\right) \tilde{\epsilon}_{0},
\end{gathered}
$$

where $\Gamma_{m}$ denotes the flat space gamma matrices (the usual Pauli matrices), i.e. $\Gamma_{m}=\left(1+\frac{x^{2}}{4 r^{2}}\right) \gamma_{m}$. The $\mathrm{SU}(2 \mid 1)_{\mathrm{A}}$ supersymmetry transformations are the restriction of (3.5) and (3.7) to these conformal Killing spinors.

- $\mathrm{SU}(2 \mid 1)_{\mathrm{B}}$. This supersymmetry algebra is obtained by restricting the conformal Killing spinors on the two-sphere (3.2) to obey

$$
\nabla_{m} \epsilon=\frac{i}{2 r} \gamma_{m} \tilde{\epsilon}, \quad \nabla_{m} \tilde{\epsilon}=\frac{i}{2 r} \gamma_{m} \epsilon,
$$

so that $\varepsilon=\epsilon_{+}+\tilde{\epsilon}_{-}$and $\bar{\varepsilon}=\tilde{\epsilon}_{+}+\epsilon_{-}$satisfy

$$
\nabla_{m} \varepsilon=\frac{i}{2 r} \gamma_{m} \varepsilon, \quad \nabla_{m} \bar{\varepsilon}=\frac{i}{2 r} \gamma_{m} \bar{\varepsilon},
$$

and

$$
\eta=\frac{i}{2 r} \tilde{\epsilon}, \quad \tilde{\eta}=\frac{i}{2 r} \epsilon
$$


The $\mathrm{SU}(2 \mid 1)_{\mathrm{B}}$ supersymmetry transformations are the restriction of (3.5) and (3.7) to these conformal Killing spinors.

- $\operatorname{OSp}(1 \mid 2)$ : massive $\mathcal{N}=(1,1)$. The superconformal $\mathcal{N}=(1,1)$ superalgebra is embedded in the superconformal $\mathcal{N}=(2,2)$ superalgebra by the restriction $\epsilon=\tilde{\epsilon}$ (and thus $\eta=\tilde{\eta}$ ). This superconformal algebra has four supercharges. It further has a massive $\operatorname{OSp}(1 \mid 2)$ subalgebra that is obtained by setting $\eta=\frac{i}{2 r} \epsilon$. This has two supercharges. The chiral and twisted chiral multiplets become reducible, each breaking up into two $\mathcal{N}=(1,1)$ real scalar multiplets.

\subsection{The $S^{2}$ partition function}

Let us now consider the $S^{2}$ partition function of $\mathcal{N}=(2,2)$ superconformal theories. Since the formal expansion (1.4) contains divergences, we need to decide which symmetries our regulator preserves. Below we will analyze the consequences of the assumption that the symmetry preserved by the regulator is one of the massive subalgebras of the $\mathcal{N}=(2,2)$ superconformal algebra discussed above.

Let us begin by assuming that the physics at coincident points is $\mathrm{SU}(2 \mid 1)_{\mathrm{A}}$ invariant. That means that we are allowed to use $\mathrm{SU}(2 \mid 1)_{\mathrm{A}}$ Ward identities, even at coincident points. With this assumption, the first observation we make is that the chiral multiplet invariant (3.5) is $\mathrm{SU}(2 \mid 1)_{\mathrm{A}}$-exact. By taking $\epsilon=0$ in (3.5) we have that

$$
F=\frac{1}{\|\tilde{\epsilon}\|^{2}} \delta\left(\tilde{\epsilon}^{\dagger} \psi\right)
$$

where $\|\tilde{\epsilon}\|^{2} \equiv \tilde{\epsilon}^{\dagger} \tilde{\epsilon}$, and $\tilde{\epsilon}$ is a nowhere vanishing Dirac conformal Killing spinor. Therefore, the $S^{2}$ partition function is independent of the chiral couplings constants. This already shows that the two-sphere partition function is not completely ambiguous. The independence of the chiral couplings implies that there is no local $\mathrm{SU}(2 \mid 1)_{\text {A }}$ supergravity counterterm that could reduce on $S^{2}$ to a function of the bottom components of the chiral couplings.

The twisted chiral multiplet invariant (3.7) is more interesting. It is not exact with respect to the $\mathrm{SU}(2 \mid 1)_{\mathrm{A}}$ subalgebra of the superconformal algebra. Indeed, in $\mathrm{SU}(2 \mid 1)_{\mathrm{A}}$ it is inconsistent to set $\tilde{\epsilon}_{-}=0$ while keeping $\tilde{\epsilon}_{+} \neq 0$, as the two chiralities are linked by the $\mathrm{SU}(2 \mid 1)_{\mathrm{A}}$ Killing spinor equation (3.8). The $\mathrm{SU}(2 \mid 1)_{\mathrm{A}}$-invariant partition function may thus depend on the twisted chiral moduli.

Even though the top component of the twisted chiral multiplet $G$ is not exact, its integral is almost so. From (3.7) we see that

$$
\delta \zeta_{+}=-i \not \nabla\left(Y \tilde{\epsilon}_{-}\right)+G \tilde{\epsilon}_{+}
$$

If $\tilde{\epsilon}_{+}$had been nowhere vanishing, then the integrated top component would have been exact. But $\tilde{\epsilon}_{+}$does vanish. Without loss of generality, we can take the zero to be at $x=0$ 
(the North Pole). This corresponds to choosing $\tilde{\epsilon}_{0+}=0$ in (3.10) so that

$$
\begin{aligned}
& \tilde{\epsilon}_{+}=\frac{i / 2 r}{\sqrt{1+\frac{x^{2}}{4 r^{2}}}} x^{m} \Gamma_{m} \tilde{\epsilon}_{0-}, \\
& \tilde{\epsilon}_{-}=\frac{1}{\sqrt{1+\frac{x^{2}}{4 r^{2}}}} \tilde{\epsilon}_{0-} .
\end{aligned}
$$

We note that the insertion of the bottom component of a twisted chiral multiplet $Y$ is invariant under this supersymmetry transformation if the operator is inserted at the North Pole of the two-sphere (and if $\epsilon_{-}$also vanishes at the North Pole).

We can focus on the physics near the point where the spinor vanishes as follows. Introducing complex coordinates $z=x^{1}+i x^{2}$ and $\bar{z}=x^{1}-i x^{2}$ (and using that $\nabla_{z} \tilde{\epsilon}_{+}=0$ and $\nabla_{z}\left(\frac{\tilde{\epsilon}_{+}^{\dagger}}{\left\|\tilde{\epsilon}_{+}\right\|^{2}}\right)=0$ ), after some simple algebra we arrive at

$$
G=\delta\left(\frac{\tilde{\epsilon}_{+}^{\dagger} \zeta_{+}}{\left\|\tilde{\epsilon}_{+}\right\|^{2}}\right)+2 i \nabla_{z}\left(\frac{1+\frac{z \bar{z}}{4 r^{2}}}{\left\|\tilde{\epsilon}_{+}\right\|^{2}} \tilde{\epsilon}_{+}^{\dagger} \Gamma_{1} \tilde{\epsilon}_{-} Y\right)
$$

This expression is only valid away from the North Pole.

We rewrite the supersymmetric invariant as

$$
\int d^{2} x \sqrt{g} G=\int_{D_{S}} d^{2} x \sqrt{g} G+\int_{D_{N}} d^{2} x \sqrt{g} G
$$

where $D_{N}=\left\{x \mid x^{2} \leq R^{2}\right\}$ and $D_{S}=\left\{x \mid x^{2} \geq R^{2}\right\}$. Using the expression (3.17), which is regular in $D_{S}$, we get

$$
\int_{D_{S}} d^{2} x \sqrt{g} G=\int_{D_{S}} d^{2} x \sqrt{g} \delta\left(\frac{\tilde{\epsilon}_{+}^{\dagger} \zeta_{+}}{\left\|\tilde{\epsilon}_{+}\right\|^{2}}\right)+2 i \int_{D_{S}} d^{2} x \sqrt{g} \nabla_{z}\left(\frac{1+\frac{z \bar{z}}{4 r^{2}}}{\left\|\tilde{\epsilon}_{+}^{\dagger}\right\|^{\dagger}} \Gamma_{1} \tilde{\epsilon}_{-} Y\right) .
$$

Inside a correlation function with other $\delta$-closed operator insertions, the first term vanishes and the entire contribution from $D_{S}$ comes from the second, total derivative term. Ultimately we will take the limit that $R \rightarrow 0$, so that the contribution from $D_{N}$ is vanishingly small. We thus need to evaluate the total derivative term in (3.19). Using polar coordinates, Stokes' theorem, and the identities

$$
\left\|\tilde{\epsilon}_{+}\right\|^{2}=\frac{1}{4 r^{2}} \frac{z \bar{z}}{1+\frac{z \bar{z}}{4 r^{2}}}\left\|\tilde{\epsilon}_{0-}\right\|^{2} \quad \tilde{\epsilon}_{+}^{\dagger} \Gamma_{1} \tilde{\epsilon}_{-}=-\frac{i}{2 r} \frac{z}{1+\frac{z \bar{z}}{4 r^{2}}}\left\|\tilde{\epsilon}_{0-}\right\|^{2},
$$

yields

$$
\int d^{2} x \sqrt{g} G=-2 r \lim _{R \rightarrow 0} \int_{0}^{2 \pi} d \theta \frac{Y(R, \theta)}{1+\frac{R^{2}}{4 r^{2}}}=-4 \pi r Y(0)=-4 \pi r Y(N) .
$$

An analogous analysis for the top component of the anti-twisted chiral multiplet yields

$$
\int d^{2} x \sqrt{g} \bar{G}=4 \pi r \bar{Y}(\infty)=4 \pi r \bar{Y}(S) .
$$


Therefore, the integrated twisted chiral (anti-twisted chiral) multiplet top component inside a correlation function inserts the bottom component of the twisted chiral (anti-twisted chiral) multiplet at the North (South) Pole of the two-sphere. We will now use these formulae to prove that $Z_{S^{2}}^{A}=\exp \left(-K_{\mathrm{tc}}\right)$.

Differentiating the partition function twice with respect to twisted chiral moduli we get

$$
\partial_{i} \partial_{\bar{j}} \log Z_{S^{2}}^{A}=\frac{1}{\pi^{2}}\left\langle\int_{S^{2}} d^{2} x \sqrt{g} G_{i}(x) \int_{S^{2}} d^{2} y \sqrt{g} \bar{G}_{\bar{j}}(y)\right\rangle .
$$

Using (3.21), (3.22) we arrive at

$$
\partial_{i} \partial_{\bar{j}} \log Z_{S^{2}}^{A}=-(4 r)^{2}\left\langle Y_{i}(N) \bar{Y}_{\bar{j}}(S)\right\rangle=-(2 r)^{4}\left\langle G_{i}(N) \bar{G}_{\bar{j}}(S)\right\rangle=-\partial_{i} \partial_{\bar{j}} K_{\mathrm{tc}} .
$$

In completing the proof we have used a supersymmetry Ward identity relating the two-point functions of the top and the bottom components of a chiral multiplet, i.e. $\left\langle G_{i}(N) \bar{G}_{\bar{j}}(S)\right\rangle=$ $\frac{1}{r^{2}}\left\langle Y_{i}(N) \bar{Y}_{\bar{j}}(S)\right\rangle$, and the fact that the metric on the conformal manifold of $\mathcal{N}=(2,2)$ superconformal field theories is Kähler, i.e. $g_{i \bar{j}}=\partial_{i} \partial_{\bar{j}} K$. Therefore, up to a holomorphic ambiguity which will be discussed in detail in the next subsection,

$$
Z_{S^{2}}^{A}=e^{-K_{\mathrm{tc}}}
$$

If we assume that the physics at coincident points is $\mathrm{SU}(2 \mid 1)_{\mathrm{B}}$ invariant, the analysis is similar to what we have done above, essentially only exchanging the role of chiral and twisted chiral multiplets. The twisted chiral invariant (3.7) is $\mathrm{SU}(2 \mid 1)_{\mathrm{B}}$-exact. The chiral

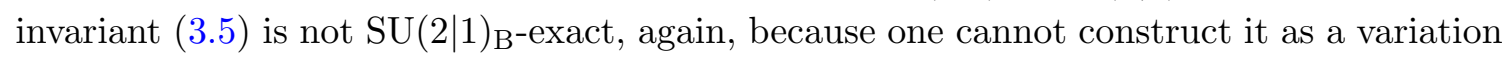
of a fermion without encountering some zero of a Killing spinor. Repeating the steps above we arrive at

$$
\partial_{i} \partial_{\bar{j}} \ln Z_{S^{2}}^{B}=-(4 r)^{2}\left\langle\phi_{i}(N) \bar{\phi}_{\bar{j}}(S)\right\rangle=-(2 r)^{4}\left\langle F_{i}(N) \bar{F}_{\bar{j}}(S)\right\rangle=-\partial_{i} \partial_{\bar{j}} K_{\mathrm{C}}
$$

Thus, up to a holomorphic ambiguity which we will discuss below,

$$
Z_{S^{2}}^{B}=e^{-K_{\mathrm{C}}} .
$$

Let us now discuss the consequences of preserving the massive $\mathcal{N}=(1,1)$ superalgebra $\operatorname{OSp}(1 \mid 2)$ described in the previous subsection. The exactly marginal operators are given by the top components of $\mathcal{N}=(1,1)$ real scalar multiplets with Weyl weight 1 . This multiplet is just the real version of the $\mathcal{N}=(2,2)$ chiral multiplet $(3.5)$, and therefore the $\mathcal{N}=(1,1)$ superconformal invariant is (3.5), but with $F$ now being real. Let us now consider the sphere partition function regulated in an $\operatorname{OSp}(1 \mid 2)$ invariant way. One can attempt to repeat the analysis performed for $\mathrm{SU}(2 \mid 1)_{\mathrm{A}}$ and $\mathrm{SU}(2 \mid 1)_{\mathrm{B}}$. However, we find that the integrated twopoint function of the top component of a real scalar multiplet does not reduce to the unintegrated two-point function of the bottom components, in contrast to the $\mathcal{N}=(2,2)$ analysis. While it is still true that the top component is not supersymmetry exact (it is of the form in (3.15)), now the bottom component of the $\mathcal{N}=(1,1)$ multiplet is not $\delta$-closed, since Dirac Killing spinors on $S^{2}$ are nowhere vanishing. Note that in our $\mathcal{N}=(2,2)$ 
derivation of (3.24) we used in a crucial way that the insertion of the bottom component of the $\mathcal{N}=(2,2)$ multiplets at the poles is $\delta$-closed under a supersymmetry transformation. In the next subsection we will construct an explicit $\mathcal{N}=(1,1)$ supergravity counterterm involving an arbitrary function of the moduli, which also sit in bottom components of $\mathcal{N}=(1,1)$ real chiral multiplets. We conclude that the partition function of $\mathcal{N}=(1,1)$ SCFTs on $S^{2}$ is scheme dependent and thus ambiguous. We will reach a very similar conclusion for the sphere partition function of four-dimensional $\mathcal{N}=1$ SCFTs in section 4 .

Before we turn to the holomorphic ambiguity afflicting the partition functions $Z_{S^{2}}^{A, B}$, let us make some comments. We discussed what happens if the theory is regulated in a manner that preserves $\mathrm{SU}(2 \mid 1)_{\mathrm{A}, \mathrm{B}}$. But what if we could find a regulator that preserves a bigger symmetry group, i.e. the full superconformal group which has eight supercharges on $S^{2}$ ? Clearly, such a regulator cannot exist. The reason is simply that if it had existed, using two different subgroups of the superconformal symmetry group, we would have arrived at two different results (3.25), (3.27). In fact, if all the eight supercharges are available to us, then one can formally prove that the chiral invariant (3.5) and the twisted chiral invariant (3.7) are both superconformal exact. Hence, one would be led to the conclusion that the partition function is independent of the exactly marginal parameters. The fact that one can get contradictory answers simply means that the full $\mathcal{N}=(2,2)$ superconformal group cannot be preserved. This is reminiscent of an anomaly.

\subsection{Kähler ambiguity counterterm and $\mathcal{N}=(1,1)$ supergravity counterterm}

The Kähler potential admits the following well-known ambiguity

$$
K(\lambda, \bar{\lambda}) \rightarrow K(\lambda, \bar{\lambda})+\mathcal{F}(\lambda)+\overline{\mathcal{F}}(\bar{\lambda}),
$$

which leaves the metric $g_{i \bar{j}}=\partial_{i} \partial_{\bar{j}} K$ invariant. The fact that the two-sphere partition function computes the Kähler potential implies that there should be a finite, local $\mathcal{N}=(2,2)$ supergravity counterterm that when evaluated on the two-sphere captures the Kähler ambiguity (3.28). For example, if we describe the same conformal field theory in two different duality frames, the partition functions may differ by some holomorphic (plus antiholomorphic) function of the moduli. This means that the partition function is a section on the conformal manifold.

We proceed to construct this local supergravity counterterm explicitly.

There are two minimal versions of $\mathcal{N}=(2,2)$ supergravity. They differ by the nature of the U(1) $R$-symmetry that is gauged (vector or axial), and are mapped into each other under the $Z_{2}$ mirror automorphism. The rigid limits of these two supergravities correspond to preserving $\mathrm{SU}(2 \mid 1)_{\mathrm{A}}$ or $\mathrm{SU}(2 \mid 1)_{\mathrm{B}}$ on the two-sphere.

We begin with the $\mathrm{SU}(2 \mid 1)_{\mathrm{B}}$ theory. The ambiguity (3.28) depends on the couplings to the exactly marginal operators from the chiral ring. They sit in background chiral multiplets of vanishing $\mathrm{U}(1)_{\mathrm{A}} R$-charge. The supergravity counterterm supersymmetrizes the product of the Ricci scalar curvature $\mathcal{R}$ with the sum of an arbitrary holomorphic function and an anti-holomorphic function of chiral multiplets

$$
\int d^{2} x \sqrt{g} \mathcal{R}\left(\mathcal{F}\left(\lambda^{i}\right)+\overline{\mathcal{F}}\left(\bar{\lambda}^{\bar{i}}\right)\right) .
$$


The relevant local counter term is obtained from the $\mathrm{SU}(2 \mid 1)_{\mathrm{B}}$ supergravity action ${ }^{7}$

$$
\int d^{2} x d^{2} \Theta \varepsilon^{-1} R \mathcal{F}\left(\Phi^{i}\right)+c . c,
$$

where $\mathcal{F}\left(\Phi^{i}\right)$ is a general holomorphic function of the chiral multiplets, $\varepsilon^{-1}$ is the chiral density superspace measure and $R$ is a chiral superfield whose bottom component is a complex scalar auxiliary field that belongs to the $\mathrm{SU}(2 \mid 1)_{\mathrm{B}}$ supergravity multiplet. The spacetime scalar curvature $\mathcal{R}$ sits in the top component of the superfield $R$. When (3.30) is evaluated in the $\mathrm{SU}(2 \mid 1)_{\mathrm{B}}$ supersymmetric two-sphere background and the superfields $\Phi^{i}$ are replaced by the constant exactly marginal parameters $\lambda^{i}$, we reproduce (3.29). This establishes the Kähler ambiguity for the $\mathrm{SU}(2 \mid 1)_{\mathrm{B}}$ theory.

Similarly, for the $\mathrm{SU}(2 \mid 1)_{\mathrm{A}}$ theory one has to consider

$$
\int d^{2} x d \Theta^{+} d \tilde{\Theta}^{-} \widehat{\varepsilon}^{-1} F \mathcal{F}\left(\Omega^{i}\right)+c . c,
$$

where $\mathcal{F}\left(\Omega^{i}\right)$ is an arbitrary holomorphic function of the twisted multiplets, and $\widehat{\varepsilon}^{-1}$ is the twisted chiral density superspace measure. $F$ is a twisted chiral superfield that contains as its lowest component a complex scalar auxiliary field that belongs to the $\mathrm{SU}(2 \mid 1)_{\mathrm{A}^{-}}$ supergravity multiplet and the scalar curvature $\mathcal{R}$ in its top component. The rigid limit of this coupling evaluated on supersymmetric backgrounds was recently considered in [37]. This supergravity counterterm evaluated in the $\mathrm{SU}(2 \mid 1)_{\text {A }}$ supersymmetric two-sphere background yields the marginal counterterm (3.29), where $\lambda^{i}$ now stand for twisted chiral exactly marginal couplings.

Hence, the finite piece of the two-sphere partition function has a holomorphic plus an anti-holomorphic ambiguity. The pieces that cannot be shifted away by a holomorphic and an anti-holomorphic function of the exactly marginal parameters are physical and calculable, as long as our regularization scheme respects $\mathrm{SU}(2 \mid 1)_{\mathrm{A}}$ or $\mathrm{SU}(2 \mid 1)_{\mathrm{B}}$, as explained in the previous subsection.

We now construct a two-dimensional $\mathcal{N}=(1,1)$ supergravity counterterm that depends on an arbitrary function of the moduli. This is an $\mathcal{N}=(1,1)$ local supersymmetrization of

$$
\int d^{2} x \sqrt{g} \mathcal{R} F\left(\lambda^{i}\right)
$$

We write down this counterterm by coupling $\mathcal{N}=(1,1)$ supergravity to real scalar multiplets $\Phi^{i}$. The moduli $\lambda^{i}$ are the bottom components of $\Phi^{i}$ while the supergravity multiplet contains the graviton, the gravitino and a real auxiliary field $B$. The superspace description of this $\mathcal{N}=(1,1)$ supergravity counterterm is

$$
\int d^{2} x d^{2} \Theta E^{-1} R F\left(\Phi^{i}\right)
$$

where $E^{-1}$ is the density measure superfield and $R$ is a real superfield, which has a complex scalar $R \mid=B$ as its bottom component and contains the scalar curvature $\mathcal{R}$ in its top

\footnotetext{
${ }^{7}$ We thank N. Seiberg for a discussion.,KetovES
} 
component [38]. This supergravity counterterm evaluated in the $\operatorname{OSp}(1 \mid 2)$ supersymmetric two-sphere background yields the marginal counterterm (3.32). Therefore, the sphere partition function of $\mathcal{N}=(1,1)$ SCFTs is not much different from the sphere partition function of nonsupersymmetric CFTs, for which the $S^{2}$ partition function does not have a preferred nontrivial continuum limit. As we shall see in section 4, we will reach the same conclusion when we study the sphere partition function of four-dimensional $\mathcal{N}=1$ SCFTs.

\subsection{An explicit regularization}

Let us for a moment forget about supersymmetry and examine a little more closely the quadratic term in the expansion (1.4) for the partition function, i.e. $\mathcal{O}=1$. This is proportional to the double integral

$$
\left\langle\int_{S^{d}} d^{d} x \sqrt{g} O_{i}(x) \int_{S^{d}} d^{d} y \sqrt{g} O_{j}(y)\right\rangle .
$$

The two-point function on $S^{d}$ can be obtained from the one in flat space by a Weyl transformation:

$$
\left\langle O_{i}(x) O_{j}(y)\right\rangle_{S^{d}}=\frac{g_{i j}}{d(x, y)^{2 \Delta}}
$$

where

$$
d(x, y)=\frac{|x-y|}{\sqrt{1+\frac{x^{2}}{4 r^{2}}} \sqrt{1+\frac{y^{2}}{4 r^{2}}}},
$$

and since the operators are exactly marginal $\Delta=d$. The integral (3.34) is ultraviolet divergent. As we have explained after (1.16), it can be regulated by setting $\Delta=d-\epsilon$. For sufficiently large and positive $\epsilon$ the integral converges and evaluates to

$$
\left\langle\int_{S^{d}} d^{d} x \sqrt{g} O_{i}(x) \int_{S^{d}} d^{d} y \sqrt{g} O_{j}(y)\right\rangle=g_{i j} \frac{\operatorname{vol}\left(S^{d}\right) \operatorname{vol}\left(S^{d-1}\right)}{2^{d+1}} \frac{\Gamma(d / 2) \Gamma(-d / 2+\epsilon)}{\Gamma(\epsilon)} .
$$

Setting $d=2$, in the $\epsilon \rightarrow 0$ limit we get that

$$
\left\langle\int_{S^{2}} d^{2} x \sqrt{g} O_{i}(x) \int_{S^{2}} d^{2} y \sqrt{g} O_{j}(y)\right\rangle=-\pi^{2} g_{i j} .
$$

In the case without any supersymmetry, this procedure of regularizing (3.34) by continuing the dimension of the operator is just one of many possible regularization schemes and since the answer is not renormalization scheme invariant, there is no particularly special meaning to $(3.38)$.

Now let us consider this regularization scheme in the context of $\mathcal{N}=(2,2)$ SCFTs. We see that (3.38) gives an incorrect answer in some situations! Indeed, we have shown above that, if we preserve $\mathrm{SU}(2 \mid 1)_{\mathrm{A}}$, the partition function is independent of the chiral couplings. However (3.38) does not distinguish the chiral from the twisted chiral couplings.

The resolution is, of course, that the procedure of analytically continuing the dimension of chiral operators is inconsistent with $\mathrm{SU}(2 \mid 1)_{\mathrm{A}}$. This is because if one changes the dimension of a chiral operator in the superpotential, one necessarily breaks $U(1)_{V}$ and therefore $\mathrm{SU}(2 \mid 1)_{\mathrm{A}}$. However, this procedure is perfectly $\mathrm{SU}(2 \mid 1)_{\mathrm{A}}$ invariant for the twisted chiral 
operators, and since we have already explained that the answer is unique, (3.38) must give the right result in this case. Indeed, (3.38) is completely consistent with the formula derived in (3.24), which implies (3.25). The mirror symmetric statement is that this regularization scheme preserves $\mathrm{SU}(2 \mid 1)_{B}$ for chiral operators while it breaks it for twisted chiral operators, and therefore leads to (3.27). We will use this derivation via analytic continuation in the conformal dimension of the exactly marginal operators again in section 5 , when we discuss four-dimensional $\mathcal{N}=2$ SCFTs.

\section{$4 \mathcal{N}=1$ field theories on $S^{4}$}

We now wish to study the dependence of the four-sphere partition function of $\mathcal{N}=1$ superconformal field theories on their exactly-marginal couplings. The exactly-marginal couplings parameterize a Kähler manifold [25], and they are commonplace in $\mathcal{N}=1$ superconformal theories [39]. These theories have a $\mathrm{U}(1)_{\mathrm{R}} \mathrm{R}$-symmetry. The exactly marginal operators of $\mathcal{N}=1$ superconformal field theories are descendants of operators in the chiral ring; they are the top component of a chiral multiplet with R-charge 2 (and Weyl weight 3 ). We note that not all such operators are exactly marginal in four-dimensional $\mathcal{N}=1$ superconformal field theories. This is unlike in unitary two-dimensional $\mathcal{N}=(2,2)$ superconformal field theories with a normalizable vacuum state, where superconformal descendant operators of the type described in the previous section are necessarily exactly marginal. ${ }^{8}$

$\mathcal{N}=1$ superconformal field theories can be canonically placed on the round foursphere. The superconformal transformations are generated by a Dirac conformal Killing spinor on $S^{4}$, which obeys

$$
\nabla_{m} \epsilon=\gamma_{m} \eta
$$

Under superconformal transformations the components of a chiral multiplet of Weyl weight $w$ (and R-charge $\left.\frac{2}{3} w\right)$ transform as [41]

$$
\begin{aligned}
\delta Z & =\frac{1}{\sqrt{2}} \bar{\epsilon} \chi_{L}, \\
\delta \chi_{L} & =\frac{1}{\sqrt{2}}\left(\not \nabla Z \epsilon_{R}+F \epsilon_{L}\right)+\sqrt{2} w Z \eta_{L}, \\
\delta F & =\frac{1}{\sqrt{2}} \bar{\epsilon} \not \chi_{L}+\sqrt{2}(1-w) \bar{\eta} \chi_{L}=\frac{1}{\sqrt{2}} \nabla_{m}\left(\bar{\epsilon} \gamma^{m} \chi_{L}\right)+\sqrt{2}(3-w) \bar{\eta} \chi_{L},
\end{aligned}
$$

where $\bar{\epsilon} \equiv \epsilon^{T} \mathcal{C}$, and $\mathcal{C}$ is the charge conjugation matrix. We have also defined chiral spinors with the projectors $P_{L}=\frac{1}{2}\left(1+\gamma_{*}\right)$ and $P_{R}=\frac{1}{2}\left(1-\gamma_{*}\right)$, so that $P_{L, R} \epsilon=\epsilon_{L, R}$.

We can construct the following $\mathcal{N}=1$ superconformal invariant using a chiral multiplet of Weyl weight $w=3$

$$
I=\int d^{4} x \sqrt{g} F .
$$

\footnotetext{
${ }^{8}$ The origin of this difference is as follows: as explained in [40], a marginal operator can cease to be exactly marginal if there is a dimension $d-2$ real multiplet that can "eat" our marginal operator and thereby become a longer multiplet. In four-dimensional $\mathcal{N}=1$ theories this is possible using conserved current multiplets. Since in two dimensions $d-2=0$, in unitary theories there is no candidate multiplet to "eat" our marginal operator. Therefore, it has to be exactly marginal.
} 
This represents a general marginal operator in the superconformal field theory. From now on, we will only discuss the exactly marginal ones. The parameters multiplying these operators, the coordinates in the conformal manifold, are realized as the lowest component of background chiral multiplets with vanishing $R$-charge.

The massive $\operatorname{OSp}(1 \mid 4)$ subalgebra of the $\mathcal{N}=1$ superconformal algebra is realized by the following conformal Killing spinors on $S^{4}$

$$
\nabla_{m} \epsilon=\frac{i}{2 r} \gamma_{m} \epsilon
$$

which corresponds to choosing

$$
\eta=\frac{i}{2 r} \epsilon
$$

The supersymmetry transformation generated by these spinors anti-commute to give the $\mathrm{SO}(5)$ isometry of the four-sphere, hence, projecting out the conformal and R-symmetry generators in the $\mathcal{N}=1$ superconformal algebra. This is why one can put massive $\mathcal{N}=1$ theories on $S^{4}$ preserving (4.4). The action of this subalgebra on the chiral multiplet (4.3) is of course induced from the action of the full superconformal group, restricted to the spinors (4.4).

One can attempt to repeat our analysis in two dimensions and aim to write the invariant (4.3) as an $\mathrm{OSp}(1 \mid 4)$ supersymmetry variation of a fermion modulo a total derivative and a zero. However, one quickly encounters a geometrical obstruction. One finds an additional term, proportional to $\left[\xi, \xi^{*}\right]_{m}$, where $\xi^{m}=\frac{1}{2} \bar{\epsilon} \gamma^{m} \epsilon$ is a complex Killing vector. This commutator cannot be put to zero on $S^{4}$ (loosely speaking, the $\operatorname{OSp}(1 \mid 4)$ algebra contains only complexified Killing vectors not commuting with their complex conjugate).

Therefore, the invariant (4.3) is not $\operatorname{OSp}(1 \mid 4)$-exact (even if one ignores zeroes of spinors and total derivatives). In fact, we now prove that the dependence of the sphere partition function of $\mathcal{N}=1$ superconformal field theories on moduli is scheme dependent. This is therefore analogous to the situation in non-supersymmetric theories.

There is a finite, local $\mathcal{N}=1$ supergravity counterterm that depends on an arbitrary real function of the chiral and anti-chiral multiplets. This counterterm once evaluated on the $\operatorname{OSp}(1 \mid 4)$ invariant four-sphere is non-vanishing and explicitly demonstrates that the dependence on the moduli of the partition function is ambiguous. Denoting chiral fields by $\Phi^{i}$ and using the conventions of [42], the relevant supergravity counterterm is

$$
-\frac{2}{3} \int d^{4} x \int d^{2} \Theta \varepsilon\left(\bar{D}^{2}-8 R\right) R \bar{R} F\left(\Phi^{i}, \bar{\Phi}^{\bar{i}}\right) .
$$

The supergravity multiplet contains, in addition to the graviton and the gravitino, two auxiliary fields - a complex scalar $M$ and a real vector $b_{\mu}$. $\varepsilon$ is the chiral superspace measure and is the superfield that contains the square root of the determinant of the metric in its bottom component. The chiral superfield $R$ has the auxiliary field $M$ as its lowest component. After setting the gravitino to zero, we get:

$$
\begin{aligned}
\varepsilon & =\frac{1}{2} \sqrt{g}-\frac{1}{2} \sqrt{g} \bar{M} \Theta^{2}, \\
R & =-\frac{1}{6} M-\frac{1}{6} \Theta^{2}\left(-\frac{1}{2} \mathcal{R}+\frac{2}{3} M \bar{M}+\frac{1}{3} b_{\mu} b^{\mu}-i \nabla_{\mu} b^{\mu}\right) .
\end{aligned}
$$


Manifolds that preserve four supercharges obey the integrability conditions [16]:

$$
\begin{aligned}
\frac{3}{2} \mathcal{R}-b_{\mu} b^{\mu}-2 M \bar{M} & =0, \\
\nabla_{\mu} b^{\mu} & =0 .
\end{aligned}
$$

Using (4.9), only the lowest component of $R$ remains. For a chiral superfield $\Omega$ with lowest component $\omega$, we have

$$
\left(\overline{\mathcal{D}}^{2}-8 R\right) \overline{R \Omega}=-\frac{2}{9} M \bar{M} \bar{\omega}+\Theta^{2}\left(\frac{2}{3} \nabla_{\mu} \partial^{\mu}(\bar{M} \bar{\omega})+\frac{4 i}{9} b_{\mu} \partial^{\mu}(\bar{M} \bar{\omega})\right)
$$

where we have set the higher components of $\Omega$ to zero since they vanish on the supersymmetric sphere (in addition, we have set the gravitino to zero).

The background fields for $S^{4}$ are $M=\bar{M}=-\frac{3 i}{r}$ and $b_{\mu}=0$, which solves (4.9). After a little algebra, we then obtain that (4.6) evaluates to

$$
\frac{1}{r^{4}} \int d^{4} x \sqrt{g} F\left(\lambda^{i}, \bar{\lambda}^{\bar{i}}\right)
$$

on the supersymmetric sphere. Thus, (4.6) supersymmetrizes the finite counterterm (4.10) for an arbitrary function of the exactly marginal parameters. This renders the sphere partition function of four-dimensional $\mathcal{N}=1$ SCFTs ambiguous.

\section{$5 \mathcal{N}=2$ field theories on $S^{4}$}

We now turn our attention to the study of the four-sphere partition function of $\mathcal{N}=2$ superconformal field theories. These theories have an $\mathrm{SU}(2)_{\mathrm{R}} \times \mathrm{U}(1)_{\mathrm{R}} \mathrm{R}$-symmetry. An exactly marginal operator in such a theory is realized as a superconformal descendant of the bottom component of a chiral multiplet of $\mathrm{U}(1)_{\mathrm{R}}$ R-charge 2 (and Weyl weight 2).

We consider now placing $\mathcal{N}=2$ superconformal field theories on $S^{4}$. The $\mathcal{N}=2$ superconformal transformations are parametrized by an $\mathrm{SU}(2)_{\mathrm{R}}$ R-symmetry doublet of left chiral conformal Killing spinors $\epsilon^{i}$ and right chiral conformal Killing spinors $\epsilon_{i}$

$$
\nabla_{m} \epsilon^{i}=\gamma_{m} \eta^{i} \quad \nabla_{m} \epsilon_{i}=\gamma_{m} \eta_{i}
$$

The supersymmetry transformations of a four dimensional $\mathcal{N}=2$ chiral multiplet of Weyl 
weight $w$ are given by [43]

$$
\begin{aligned}
\delta A & =\frac{1}{2} \bar{\epsilon}^{i} \Psi_{i}, \\
\delta \Psi_{i} & =\not A \epsilon_{i}+\frac{1}{2} B_{i j} \epsilon^{j}+\frac{1}{4} \gamma^{a b} F_{a b}^{-} \varepsilon_{i j} \epsilon^{j}+2 w A \eta_{i} \\
& =\not\left(A \epsilon_{i}\right)+\frac{1}{2} B_{i j} \epsilon^{j}+\frac{1}{4} \gamma^{a b} F_{a b}^{-} \varepsilon_{i j} \epsilon^{j}+(2 w-4) A \eta_{i} \\
\delta B_{i j} & =\bar{\epsilon}_{(i} \not \nabla \Psi_{j}-\bar{\epsilon}^{k} \Lambda_{(i} \varepsilon_{j) k}+2(1-w) \bar{\eta}_{(i} \Psi_{j}, \\
\delta F_{a b}^{-} & =\frac{1}{4} \varepsilon^{i j} \bar{\epsilon}_{i} \not \nabla \gamma_{a b} \Psi_{j}+\frac{1}{4} \bar{\epsilon}^{i} \gamma_{a b} \Lambda_{i}-\frac{1}{2}(1+w) \varepsilon^{i j} \bar{\eta}_{i} \gamma_{a b} \Psi_{j}, \\
\delta \Lambda_{i} & =-\frac{1}{4} \gamma^{a b} \not \nabla F_{a b}^{-} \epsilon_{i}-\frac{1}{2} \not \nabla B_{i j} \varepsilon^{j k} \epsilon_{k}+\frac{1}{2} C \varepsilon_{i j} \epsilon^{j}-(1+w) B_{i j} \varepsilon^{j k} \eta_{k}+\frac{1}{2}(1-w) \gamma^{a b} F_{a b}^{-} \eta_{i} \\
& =-\frac{1}{4} \gamma^{a b} \not \nabla\left(F_{a b}^{-} \epsilon_{i}\right)-\frac{1}{2} \not \nabla B_{i j} \varepsilon^{j k} \epsilon_{k}+\frac{1}{2} C \varepsilon_{i j} \epsilon^{j}-(1+w) B_{i j} \varepsilon^{j k} \eta_{k}+\frac{1}{2}(3-w) \gamma^{a b} F_{a b}^{-} \eta_{i}, \\
\delta C & =-\varepsilon^{i j} \bar{\epsilon}_{i} \not \nabla \Lambda_{j}+2 w \varepsilon^{i j} \bar{\eta}_{i} \Lambda_{j}=-\nabla_{m}\left(\varepsilon^{i j} \bar{\epsilon}_{i} \gamma^{m} \Lambda_{j}\right)+(2 w-4) \varepsilon^{i j} \bar{\eta}_{i} \Lambda_{j},
\end{aligned}
$$

where as in the previous section $\bar{\lambda}=\lambda^{T} \mathcal{C}$, where $\mathcal{C}$ is the charge conjugation matrix. The $\mathcal{N}=2$ superconformal invariant realizing an exactly marginal operator is constructed from such a multiplet of Weyl weight 2. Indeed, it follows from (5.2) that

$$
I=\int d^{4} x \sqrt{g} C
$$

is invariant, where $C$ is the top component of the multiplet (5.2).

The complex parameters that span the conformal manifold and source the $\mathcal{N}=2$ exactly marginal operators are realized as the bottom components of $\mathcal{N}=2$ chiral multiplets of Weyl weight 0 . Note that Weyl weight 0 chiral superfields are irreducible [44], thus, in general, we cannot embed the exactly marginal couplings in $\mathcal{N}=2$ vector multiplets.

We are interested in unraveling the physical content of the sphere partition function of $\mathcal{N}=2$ SCFTs. We consider regulating the ultraviolet divergences in an $\operatorname{OSp}(2 \mid 4)$ invariant fashion. This is the $\mathcal{N}=2$ supersymmetry algebra of a massive theory on the foursphere $S^{4}$. We describe it below in detail. We will prove that the $S^{4}$ partition function of $\mathcal{N}=2$ superconformal field theories regulated in an $\operatorname{OSp}(2 \mid 4)$ invariant fashion computes the Kähler potential on the conformal manifold

$$
Z_{S^{4}}=e^{K / 12} .
$$

The $\operatorname{OSp}(2 \mid 4)$ supersymmetry transformations are generated by conformal Killing spinors $\epsilon^{j}$ and $\epsilon_{j}$ obeying

$$
\nabla_{m} \epsilon^{j}=\frac{i}{2 r} \gamma_{m} \tau_{1}^{j k} \epsilon_{k} \quad \nabla_{m} \epsilon_{j}=\frac{i}{2 r} \gamma_{m} \tau_{1 j k} \epsilon^{k}
$$

where $\tau_{1}^{j k}=i \sigma_{3}^{j k}=\left(\tau_{1 j k}\right)^{*}$, and $\sigma_{3}=\left(\begin{array}{cc}1 & 0 \\ 0 & -1\end{array}\right) \cdot{ }^{9}$ These correspond to conformal Killing spinors (5.1) with

$$
\eta^{j}=\frac{i}{2 r} \tau_{1}^{j k} \epsilon_{k} \quad \eta_{j}=\frac{i}{2 r} \tau_{1 j k} \epsilon^{k} .
$$

\footnotetext{
${ }^{9}$ We introduce this notation in order to follow the notations of [41].
} 
We can diagonalize equations (5.5) by defining

$$
\chi^{j}=\epsilon^{j}+\tau_{1}^{j k} \epsilon_{k} \quad \hat{\chi}^{i}=\epsilon^{j}-\tau_{1}^{j k} \epsilon_{k}
$$

so that

$$
\nabla_{m} \chi^{j}=\frac{i}{2 r} \gamma_{m} \chi^{j}
$$

and $\widehat{\chi}^{j}=\gamma_{*} \chi^{j}$, where $P_{L / R}=\frac{1}{2}\left(1 \pm \gamma_{*}\right)$.

Computing the commutator of the $\mathcal{N}=2$ superconformal transformations (5.2) acting on the fields of a chiral multiplet gives a representation of the $\mathcal{N}=2$ superconformal algebra on the fields

$$
\left[\delta_{1}, \delta_{2}\right]=\xi^{m} P_{m}+\lambda_{a} R^{a}+\lambda_{\mathrm{D}} D+\lambda_{R} R+\frac{1}{2} \lambda^{a b} L_{a b} .
$$

With the choice of spinors (5.5), it is easy to prove that the vector field produced by two superconformal transformations

$$
\xi_{m}=\frac{1}{2} \bar{\epsilon}_{2}^{i} \gamma_{m} \epsilon_{1 i}+\frac{1}{2} \bar{\epsilon}_{2 i} \gamma_{m} \epsilon_{1}^{i}
$$

is a Killing vector on $S^{4}$. Moreover, with the choice of spinors (5.5) the parameters associated to local dilatations and $\mathrm{U}(1)_{\mathrm{R}} \mathrm{R}$-symmetry vanish, while the $\mathrm{SU}(2)_{\mathrm{R}} \mathrm{R}$-symmetry is broken down to $\mathrm{SO}(2)_{\mathrm{R}}$

$$
\begin{aligned}
\lambda_{\mathrm{D}} & =-\frac{1}{2}\left(\bar{\epsilon}_{1}^{i} \eta_{2 i}+\bar{\epsilon}_{1 i} \eta_{2}^{i}-\bar{\epsilon}_{2}^{i} \eta_{1 i}-\bar{\epsilon}_{2 i} \eta_{1}^{i}\right)=0, \\
\lambda_{R} & =\frac{i}{2}\left(\bar{\epsilon}_{1}^{i} \eta_{2 i}-\bar{\epsilon}_{1 i} \eta_{2}^{i}-\bar{\epsilon}_{2}^{i} \eta_{1 i}+\bar{\epsilon}_{2 i} \eta_{1}^{i}\right)=0, \\
\lambda_{a} & =i\left(-\bar{\epsilon}_{1}^{i} \eta_{2 j}+\bar{\epsilon}_{1 j} \eta_{2}^{i}+\bar{\epsilon}_{2}^{i} \eta_{1 j}-\bar{\epsilon}_{2 j} \eta_{1}^{i}\right) \sigma_{a i}{ }^{j} \Longrightarrow \lambda_{1}=\frac{i}{r} \varepsilon_{i j} \bar{\chi}_{1}^{i} \chi_{2}^{j}, \quad \lambda_{2}=\lambda_{3}=0 .
\end{aligned}
$$

Here $\sigma_{a}$ are the Pauli matrices. Thus the Killing spinors generate the $\operatorname{OSp}(2 \mid 4)$ supersymmetry algebra, whose supercharges close into an $\mathrm{SO}(5)$ isometry and an $\mathrm{SO}(2)_{\mathrm{R}} \mathrm{R}$ symmetry. ${ }^{10}$ This is the symmetry of a general massive $\mathcal{N}=2$ theory on $S^{4}$.

Using the $\operatorname{OSp}(2 \mid 4)$ supersymmetry transformations of a chiral multiplet (5.2) we expect that the top component $C$ of the multiplet with Weyl weight 2 can be written as three consecutive supersymmetry transformations of a linear combination of fermions in the multiplet modulo a zero and a total derivative. One could then repeat the argument of section 3 and arrive at (5.4). Instead, here we follow a closely related strategy, extending to four dimensions the localization proof of (5.4) presented in [15]. In addition, we derive (5.4) by an explicit supersymmetric regularization. The two derivations agree.

\subsection{The Kähler potential from the four-sphere}

First, we employ the localization computation [26] of the $S^{4}$ partition function of Lagrangian four dimensional $\mathcal{N}=2$ superconformal field theories. These theories are based

\footnotetext{
${ }^{10}$ On the fields, a local Lorentz transformation with parameter $\lambda^{a b}=-\nabla^{[a} \xi^{b]}$ is also induced.
} 
on vector multiplets with gauge group $G$ and hypermultiplets transforming in a representation $R$ of $G$. The partition function can be computed by localizing the functional integral with respect to a supercharge in $\operatorname{OSp}(2 \mid 4)$. For our analysis, the details of the hypermultiplets, which vanish on the localization saddle points [26], are not important. Therefore, we can focus on the $\mathcal{N}=2$ vector multiplets.

The $\mathcal{N}=2$ supersymmetric vector multiplet action is constructed from the top component of an $\mathcal{N}=2$ chiral and anti-chiral multiplet of Weyl weight 2

$$
A_{i}=\frac{i}{2} F\left(\Phi_{i}\right)=\frac{i}{8 \pi} \operatorname{Tr} \Phi_{i}^{2} \quad \bar{A}_{i}=-\frac{i}{2} \bar{F}\left(\bar{\Phi}_{i}\right)=-\frac{i}{8 \pi} \operatorname{Tr} \bar{\Phi}_{i}^{2}
$$

where $\Phi_{i}$ is an $\mathcal{N}=2$ vector multiplet of Weyl weight 1 associated to the gauge group factor $G_{i}{ }^{11}$ The four-sphere $\mathcal{N}=2$ supersymmetric vector multiplet action is

$$
S=\int d^{4} x \sqrt{g} \sum_{i}\left(\tau_{i} C_{i}+\bar{\tau}_{\bar{i}} \bar{C}_{\bar{i}}\right)
$$

where $C_{i}$ and $\bar{C}_{\bar{i}}$ are the top components of the composite chiral and anti-chiral multiplets. The exactly marginal parameters are the complexified gauge couplings

$$
\tau_{i}=\frac{\theta_{i}}{2 \pi}+\frac{4 \pi i}{g_{i}^{2}} .
$$

Calculating the second derivative with respect to the marginal couplings we get

$$
\partial_{i} \partial_{\bar{j}} \log Z_{S^{4}}=\frac{1}{\pi^{4}}\left\langle\int_{S^{4}} d^{4} x \sqrt{g} C_{i}(x) \int_{S^{4}} d^{4} y \sqrt{g} \bar{C}_{\bar{j}}(y)\right\rangle=\left(32 r^{2}\right)^{2}\left\langle A_{i}(N) \bar{A}_{\bar{j}}(S)\right\rangle .
$$

In the final step, we have used supersymmetric localization.

To relate this to the Zamolodchikov metric we can use the supersymmetry transformations (5.2) to relate the two-point function of the bottom components (5.15) to the two-point function of the top components. This yields $\left\langle A_{i}(N) \bar{A}_{\bar{j}}(S)\right\rangle=\frac{r^{4}}{48}\left\langle C_{i}(N) \bar{C}_{\bar{j}}(S)\right\rangle$. Finally, we need to relate the two-point function $\left\langle C_{i}(N) \bar{C}_{\bar{j}}(S)\right\rangle$ to the Zamolodchikov metric. The result is $\left\langle C_{i}(N) \bar{C}_{\bar{j}}(S)\right\rangle=\frac{1}{(2 r)^{8}} g_{i \bar{j}}$. Combining all the factors we find

$$
\partial_{i} \partial_{\bar{j}} \log Z_{S^{4}}=\frac{1}{12} g_{i \bar{j}}=\frac{1}{12} \partial_{i} \partial_{\bar{j}} K .
$$

This shows $(2.5)$

$$
Z_{S^{4}}=e^{K / 12}
$$

We can now compare this result to another derivation. In section 3, equation (3.37), we have evaluated the integrated two-point function using regularization by analytic continuation in the scaling dimension. As we have explained in section 3, this regularization does not always work. One needs to make sure that it preserves the massive supersymmetry algebra. In the context of $\mathcal{N}=2$ on $S^{4}$, this is indeed the case, because one can vary the

\footnotetext{
${ }^{11}$ The argument below can be carried without referring to a specific microscopic realization. This requires solving for the supersymmetric configurations of the $\mathcal{N}=2$ chiral multiplet of Weyl weight 2 . To simplify the analysis we refer to the elementary fields $\Phi_{i}$.
} 
scaling dimension of a chiral operator without breaking the $\mathrm{SO}(2)_{\mathrm{R}} \subset \mathrm{SU}(2)_{\mathrm{R}}$ symmetry. Plugging $d=4$ in (3.37) and taking the limit $\epsilon \rightarrow 0$, we find precisely (5.16) (after correctly normalizing the operators, as in (1.1)). This therefore provides a derivation of (5.16) that does not depend on localization.

Let us discuss a simple example, that of $\mathcal{N}=4$ super-Yang-Mills on the four-sphere. The conformal manifold has one complex parameter that preserves $\mathcal{N}=4$ supersymmetry, i.e. the gauge coupling. The $S^{4}$ partition function depends on the masses of the fields in the adjoint hypermultiplet, and those need to be tuned such that the four-sphere partition function is that of the conformally coupled theory. For the correct, conformal choice of the mass parameter [45], the instanton contributions to the four-sphere partition function vanish, and one finds via our prescription (5.17) $K \sim \log (i(\bar{\tau}-\tau))$. If we perform an $\operatorname{SL}(2, \mathrm{Z})$ $S$-duality transformation, the Kähler potential shifts by $\log (\tau)+\log (\bar{\tau})+$ const. This is a manifestation of a fact that we have explained in detail in section 3 ; the partition function is a section rather than a function. This Kähler transformation should be understood as a local supergravity counterterm, similar to the ones we have found in section 3 . We leave this as well as the study of the Kähler geometry in more general $\mathcal{N}=2$ examples for the future.

\section{Acknowledgments}

We are very grateful to C. Closset, L. Di Pietro, N. Doroud, T. Dumitrescu, B. Le Floch, D. Gaiotto, S. Lee, J. Maldacena, V. Narovlansky, J. Polchinski, A. Schwimmer, A. Van Proeyen for useful discussions. We especially thank N. Seiberg for helpful discussions at various stages of the project. JG and ZK are grateful to the KITP for its warm hospitality during the initial stages of this project, which was supported in part by the National Science Foundation under Grant No. NSF PHY11-25915. EG and ZK thank the Perimeter Institute for its very kind hospitality during the course of this project. Research at the Perimeter Institute is supported in part by the Government of Canada through NSERC and by the Province of Ontario through MRI. J.G. also acknowledges further support from an NSERC Discovery Grant and from an ERA grant by the Province of Ontario. ZK is supported by the ERC STG grant number 335182, by the Israel Science Foundation under grant number 884/11, by the United States-Israel Binational Science Foundation (BSF) under grant number 2010/629, and by the I-CORE Program of the Planning and Budgeting Committee and by the Israel Science Foundation under grant number 1937/12. Any opinions, findings, and conclusions or recommendations expressed in this material are those of the authors and do not necessarily reflect the views of the funding agencies.

Open Access. This article is distributed under the terms of the Creative Commons Attribution License (CC-BY 4.0), which permits any use, distribution and reproduction in any medium, provided the original author(s) and source are credited. 


\section{References}

[1] A.B. Zamolodchikov, Irreversibility of the Flux of the Renormalization Group in a $2 D$ Field Theory, JETP Lett. 43 (1986) 730 [INSPIRE].

[2] D. Kutasov, Geometry on the Space of Conformal Field Theories and Contact Terms, Phys. Lett. B 220 (1989) 153 [InSPIRE].

[3] S. Deser and A. Schwimmer, Geometric classification of conformal anomalies in arbitrary dimensions, Phys. Lett. B 309 (1993) 279 [hep-th/9302047] [INSPIRE].

[4] J.L. Cardy, Is There a c Theorem in Four-Dimensions?, Phys. Lett. B 215 (1988) 749 [INSPIRE].

[5] I. Jack and H. Osborn, Analogs for the c Theorem for Four-dimensional Renormalizable Field Theories, Nucl. Phys. B 343 (1990) 647 [InSPIRE].

[6] Z. Komargodski and A. Schwimmer, On Renormalization Group Flows in Four Dimensions, JHEP 12 (2011) 099 [arXiv:1107.3987] [INSPIRE].

[7] Z. Komargodski, The Constraints of Conformal Symmetry on RG Flows, JHEP 07 (2012) 069 [arXiv: 1112.4538] [INSPIRE].

[8] E. Witten, Quantum Field Theory and the Jones Polynomial, Commun. Math. Phys. 121 (1989) 351 [INSPIRE].

[9] C. Closset, T.T. Dumitrescu, G. Festuccia, Z. Komargodski and N. Seiberg, Contact Terms, Unitarity and F-Maximization in Three-Dimensional Superconformal Theories, JHEP 10 (2012) 053 [arXiv: 1205.4142] [INSPIRE].

[10] C. Closset, T.T. Dumitrescu, G. Festuccia, Z. Komargodski and N. Seiberg, Comments on Chern-Simons Contact Terms in Three Dimensions, JHEP 09 (2012) 091 [arXiv:1206.5218] [INSPIRE].

[11] H. Casini, M. Huerta and R.C. Myers, Towards a derivation of holographic entanglement entropy, JHEP 05 (2011) 036 [arXiv: 1102.0440] [INSPIRE].

[12] D.L. Jafferis, I.R. Klebanov, S.S. Pufu and B.R. Safdi, Towards the F-Theorem: $N=2$ Field Theories on the Three-Sphere, JHEP 06 (2011) 102 [arXiv:1103.1181] [INSPIRE].

[13] H. Casini and M. Huerta, On the RG running of the entanglement entropy of a circle, Phys. Rev. D 85 (2012) 125016 [arXiv:1202.5650] [INSPIRE].

[14] H. Jockers, V. Kumar, J.M. Lapan, D.R. Morrison and M. Romo, Two-Sphere Partition Functions and Gromov-Witten Invariants, Commun. Math. Phys. 325 (2014) 1139 [arXiv:1208.6244] [INSPIRE].

[15] J. Gomis and S. Lee, Exact Kähler Potential from Gauge Theory and Mirror Symmetry, JHEP 04 (2013) 019 [arXiv: 1210.6022] [INSPIRE].

[16] G. Festuccia and N. Seiberg, Rigid Supersymmetric Theories in Curved Superspace, JHEP 06 (2011) 114 [arXiv: 1105.0689] [InSPIRE].

[17] N. Doroud, J. Gomis, B. Le Floch and S. Lee, Exact Results in D=2 Supersymmetric Gauge Theories, JHEP 05 (2013) 093 [arXiv: 1206.2606] [InSPIRE].

[18] F. Benini and S. Cremonesi, Partition functions of $N=(2,2)$ gauge theories on $S^{2}$ and vortices, arXiv: 1206.2356 [INSPIRE]. 
[19] N. Doroud and J. Gomis, Gauge theory dynamics and Kähler potential for Calabi-Yau complex moduli, JHEP 1312 (2013) 99 [arXiv:1309.2305] [INSPIRE].

[20] S.V. Ketov, 2-d, $N=2$ and $N=4$ supergravity and the Liouville theory in superspace, Phys. Lett. B 377 (1996) 48 [hep-th/9602038] [INSPIRE].

[21] D.S. Park and J. Song, The Seiberg-Witten Kähler Potential as a Two-Sphere Partition Function, JHEP 01 (2013) 142 [arXiv:1211.0019] [INSPIRE].

[22] G. Bonelli, A. Sciarappa, A. Tanzini and P. Vasko, Vortex partition functions, wall crossing and equivariant Gromov-Witten invariants, arXiv:1307.5997 [INSPIRE].

[23] W.A. Bardeen and B. Zumino, Consistent and Covariant Anomalies in Gauge and Gravitational Theories, Nucl. Phys. B 244 (1984) 421 [INSPIRE].

[24] C.G. Callan Jr. and J.A. Harvey, Anomalies and Fermion Zero Modes on Strings and Domain Walls, Nucl. Phys. B 250 (1985) 427 [InSPIRE].

[25] V. Asnin, On metric geometry of conformal moduli spaces of four-dimensional superconformal theories, JHEP 09 (2010) 012 [arXiv:0912.2529] [INSPIRE].

[26] V. Pestun, Localization of gauge theory on a four-sphere and supersymmetric Wilson loops, Commun. Math. Phys. 313 (2012) 71 [arXiv:0712.2824] [INSPIRE].

[27] S. Cecotti and C. Vafa, Topological antitopological fusion, Nucl. Phys. B 367 (1991) 359 [INSPIRE].

[28] K. Papadodimas, Topological Anti-Topological Fusion in Four-Dimensional Superconformal Field Theories, JHEP 08 (2010) 118 [arXiv:0910.4963] [INSPIRE].

[29] B. de Wit, S. Katmadas and M. van Zalk, New supersymmetric higher-derivative couplings: Full $N=2$ superspace does not count!, JHEP 01 (2011) 007 [arXiv:1010.2150] [INSPIRE].

[30] D. Butter, B. de Wit, S.M. Kuzenko and I. Lodato, New higher-derivative invariants in $N=2$ supergravity and the Gauss-Bonnet term, JHEP 12 (2013) 062 [arXiv:1307.6546] [INSPIRE].

[31] N. Seiberg, Observations on the Moduli Space of Superconformal Field Theories, Nucl. Phys. B 303 (1988) 286 [INSPIRE].

[32] S. Cecotti, S. Ferrara and L. Girardello, Geometry of Type II Superstrings and the Moduli of Superconformal Field Theories, Int. J. Mod. Phys. A 4 (1989) 2475 [inSPIRE].

[33] A. Strominger, Special geometry, Commun. Math. Phys. 133 (1990) 163 [INSPIRE].

[34] N.C. Bizet, A. Klemm and D.V. Lopes, Landscaping with fluxes and the E8 Yukawa Point in F-theory, arXiv: 1404.7645 [INSPIRE].

[35] H. Osborn, Weyl consistency conditions and a local renormalization group equation for general renormalizable field theories, Nucl. Phys. B 363 (1991) 486 [INSPIRE].

[36] W. Lerche, C. Vafa and N.P. Warner, Chiral Rings in $N=2$ Superconformal Theories, Nucl. Phys. B 324 (1989) 427 [INSPIRE].

[37] C. Closset and S. Cremonesi, Comments on $\mathcal{N}=(2,2)$ supersymmetry on two-manifolds, JHEP 07 (2014) 075 [arXiv: 1404.2636] [INSPIRE].

[38] S.J. Gates Jr., Ectoplasm has no topology, Nucl. Phys. B 541 (1999) 615 [hep-th/9809056] [INSPIRE]. 
[39] R.G. Leigh and M.J. Strassler, Exactly marginal operators and duality in four-dimensional $N=1$ supersymmetric gauge theory, Nucl. Phys. B 447 (1995) 95 [hep-th/9503121] [INSPIRE].

[40] D. Green, Z. Komargodski, N. Seiberg, Y. Tachikawa and B. Wecht, Exactly Marginal Deformations and Global Symmetries, JHEP 06 (2010) 106 [arXiv:1005.3546] [INSPIRE].

[41] D.Z. Freedman and A. Van Proeyen, Supergravity, Cambridge, U.K., Cambridge Univ. Pr., 2012, pg. 607.

[42] J. Wess and J. Bagger, Supersymmetry and supergravity, Princeton, U.S.A., Univ. Pr., 1992, pg. 259.

[43] A. Van Proeyen, $\mathcal{N}=2$ Supergravity in $d=4,5,6$ and its Matter Couplings, http://itf.fys.kuleuven.be/ toine/LectParis.pdf.

[44] M. de Roo, J.W. van Holten, B. de Wit and A. Van Proeyen, Chiral Superfields in $N=2$ Supergravity, Nucl. Phys. B 173 (1980) 175 [InSPIRE].

[45] T. Okuda and V. Pestun, On the instantons and the hypermultiplet mass of $N=2^{*}$ super Yang-Mills on $S^{4}$, JHEP 03 (2012) 017 [arXiv: 1004.1222] [INSPIRE]. 